\title{
Exploration of uncertainty in bidirectional vector fields
}

\author{
Torre Zuk $^{a b}$, Jon Downton ${ }^{b}$, David Gray ${ }^{b}$, Sheelagh Carpendale ${ }^{a}$, and JD Liang ${ }^{b}$ \\ ${ }^{a}$ Department of Computer Science, University of Calgary, Calgary, Canada \\ ${ }^{b}$ CGGVeritas
}

\begin{abstract}
While their importance is increasingly recognized, there remain many challenges in the development of uncertainty visualizations. We introduce two uncertainty visualizations for 2D bidirectional vector fields: one based on a static glyph and the other based on animated flow. These visualizations were designed for the task of understanding and interpreting anisotropic rock property models in the domain of seismic data processing. Aspects of the implementations are discussed relating to design, interaction, and tasks.
\end{abstract}

Keywords: Uncertainty, visualization, vector field, flow, interactivity.

\section{INTRODUCTION}

While visualizing both the data and its associated uncertainty has been accepted as beneficial for accurate interpretation, the integration of uncertainty information into an existing or new visualization is not standard practice. The practical tasks of maintaining ease of comprehension for both the data and the uncertainty are not straight forward. Hence, in building uncertainty visualizations there still exist many challenges, such as finding good representations of errors and uncertainty for $3 \mathrm{D}$ visualizations ${ }^{9}$ and understanding how knowledge of information uncertainty influences analysis. ${ }^{14}$ As a result, even choosing an initial design may be difficult.

We provide two new visualizations for bidirectional vector fields with their associated uncertainty. With similar goals to Wittenbrink et al.'s work with vector fields ${ }^{23}$ we also provide a new uncertainty glyph. The new glyph provides bidirectionality and uncertainty information for orientation and magnitude in a dense field. We describe the interactive control over both the form and presence of the glyphs that are created on slice planes, or surfaces, in a 3D volume.

Building upon the image/texture based flow visualizations of van $\mathrm{Wijk}^{21}$ and Jobard et al. ${ }^{8}$ and uncertainty extensions of Botchen et al. ${ }^{3}$ we allow user driven exploration of the uncertainty in directionality, orientation, and magnitude. Our method utilizes GPU programming to achieve interactive flow visualizations and intuitively handles the ambiguity of bidirectionality as well as orientation and magnitude. We provide a probe for interactive querying of the flow field. This affords user controllable directionality enabling a visualization of possible realizations while at the same time revealing their certainty. Examples showing short animation sequences of both of our types of visualizations are shown in Figures 1 and 2 .

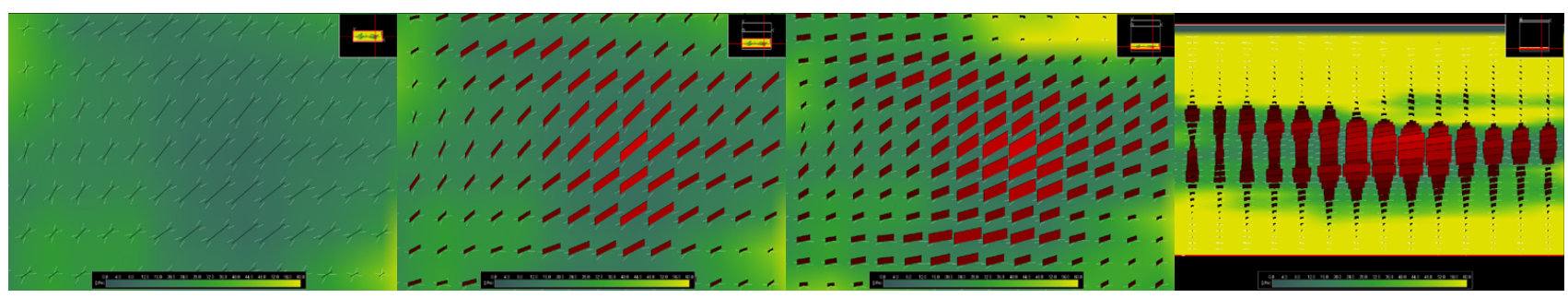

Figure 1. Sequence showing the rotation of a slice plane containing uncertainty glyphs from a top view (left) to a side view (right). Inset in the top right corners show the contextual overview of the slice in the $3 \mathrm{D}$ volume.

Further author information: (Send correspondence to T.Z.)

T.Z.: E-mail: zuk@cpsc.ucalgary.ca

Visualization and Data Analysis 2008, edited by Katy Börner,

Matti T. Gröhn, Jinah Park, and Johnathan C. Roberts, Proc. of SPIE-IS\&T

Electronic Imaging, SPIE Vol. 6809, 68090B (C)2008 SPIE and IS\&T

This is made available as an electronic reprint with permission of SPIE and IS\&T.

One print or electronic copy may be made for personal use only. Systematic

or multiple reproduction, distribution to multiple locations via electronic

or other means, duplication of any material in this paper for a fee or for

commercial purposes, or modification of the content of the paper are prohibited. 


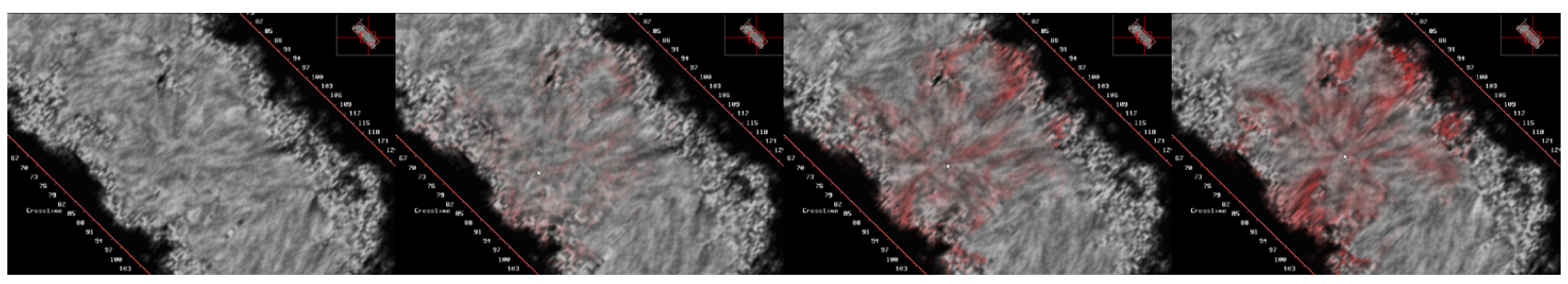

Figure 2. Animation showing flow direction query probe (cursor) with uncertainty in direction revealed using dye (from left to right). Flow is redirected directly to cursor if within uncertainty range.

\section{RELATED WORK}

In this section we will briefly summarize other work on uncertainty visualization in general, and for vector fields and flow that are related to our implementations. Some previous evaluation work related to vector fields is also provided, as it is important to consider task performance in designing any visualization in addition to thinking about potential evaluation methods.

\subsection{Uncertainty Visualization}

Uncertainty can be approached in many ways, from a focus on the data and representation, ${ }^{15}$ to analyzing the types of uncertainty, ${ }^{19}$ and even from the standpoint of reasoning. ${ }^{26}$ MacEachren proposed the use of colour saturation and blurring as being conducive to indicate uncertainty. ${ }^{13}$ These attributes may intuitively be more natural encodings but their superiority to other encodings remains to be proven. Various encodings of uncertainty are possible and user tasks should likely be a major influence in selecting one. ${ }^{25}$ Encoding single or multiple types of uncertainty in a way that can enhance interpretation is still a difficult problem.

Numerous uncertainty visualizations have been proposed for different domains, data, and types of uncertainty, 3,7,12, 15, 16 but we still require improved understanding of what makes a good uncertainty visualization. ${ }^{14}$ In the field of GIS, frameworks have been proposed to guide displaying error and uncertainty, such as Beard and Buttenfield's ${ }^{2}$ suggestions for mapping error analysis method to graphical display; however the general applicability of these frameworks is not clear. Exposing uncertainty and showing the possible effect of this uncertainty on outcomes is one of Amar and Stasko's ${ }^{1}$ design and evaluation precepts for information visualization, and is applicable to visualization in general.

\subsection{Vector Fields and Flow}

As vector fields can be used to create flow fields, flow may be a natural representation as it less abstract. Providing both abstract and realistic representations may benefit users who have trouble conceptualizing the model. Various methods for visualizing the uncertainties in flow and vector fields have been proposed. Adding uncertainty into a visualization complicates the information decoding process for the user, as the additional data is not an independent variate. Similarly, the task is also changed in that it may call for the weighing or modification of interpretations of the visualization without uncertainty. Wittenbrink et al. introduced what they called verity visualizations ${ }^{23}$ as a design recommendation for vector field uncertainty glyphs. Verity visualizations used representations of uncertainty integrated with the data without the use of additional graphic variables (colour, value, ...). This design provided vector glyphs that holistically show uncertainty in magnitude and orientation.

Wittenbrink et al.'s $\mathrm{s}^{23}$ evaluation showed the verity uncertainty glyph could be decoded with similar error to an arrow glyph decoding, and allowed for uncertainty information decoding (with comparable error). Laidlaw et al. ${ }^{10}$ evaluated static 2D vector field visualizations based on three tasks from fluid mechanics: locating critical points, identifying their type, and predicting particle advection. Their study compared time and error measures for six different static visualizations: grid and jittered arrow placement, icons with artistic layering, line integral convolution (LIC), image-guided streamlines/integral curves, and streamlines seeded on a regular grid. They found superiority in image-guided streamlines for advection tasks, LIC for location tasks, and streamlines seeded on a regular grid for critical point classification. While the LIC performed at the top for location tasks it was at or near the bottom for the advection and critical point type determination tasks, probably due to the ambiguity of direction. ${ }^{10}$ As the other five visualization types had direction encoded we feel that a more fair 
comparison would have been against animated flow, although comparing static and animated methods has its own set of problems. However, we hypothesize animated flow (e.g. streak-lines) could potentially be at the top for all their measures.

Lodha et al. ${ }^{11}$ provided a visualization system for the uncertainties found in flow fields. Variations included glyphs, envelopes of trajectories, and other representations from a stream-line or particle point of view. Sanderson et al. ${ }^{17}$ created a method for visualizing vector fields while potentially encoding uncertainty by using a reaction-diffusion model. Animated image-based flow ${ }^{21}$ has been extended to multiple scales to provide levels of detail by Telea and Strzodka. ${ }^{18}$ Botchen et al. ${ }^{3}$ introduced some novel variations of cross-advection/diffusion and multi-frequency noise to depict the uncertainty in flow. The uncertainty visualizations we introduce for bidirectional vector fields follow from this previous work on glyphs and flow representations.

\section{SEISMIC DOMAIN: DATA AND UNCERTAINTY}

This case study deals with data and tasks relevant to the seismic industry which we will briefly introduce. Seismic wave azimuthal amplitude variation versus angle of incidence has proven to be useful in characterizing fracture distributions and direction for hydrocarbon reservoirs. Downton and Gray ${ }^{5}$ describe a Bayesian process for determining the geological model parameters of anisotropy gradient $\left(B_{a n i}\right)$ and horizontal transverse isotropy symmetry orientation $\left(\Phi_{i s o}\right)$, which are related to rock fracture density and orientation respectively.

The general process to estimate these parameters is driven by the changes in seismic wave amplitude variation over multiple orientations versus the wave incidence angle on a reflector. In their process the determination of uncertainty in $B_{a n i}$ and $\Phi_{i s o}$ requires joint probability distributions to be marginalized based on the integrals

$$
P\left(B_{\text {ani }}\right)=\int_{0}^{2 \pi} g\left(B_{\text {ani }}, \Phi_{i s o}\right) d \Phi_{\text {iso }}
$$

and

$$
P\left(\Phi_{i s o}\right)=\int_{0}^{2 \pi} g\left(B_{\text {ani }}, \Phi_{i s o}\right) d B_{\text {ani }} .
$$

As it is only possible to evaluate these integrals analytically for a number of special cases, the integrals were evaluated using a numerical approximation. After further transformations the marginalized distributions are approximately Gaussian in shape as can be seen in Figure 3 and thus provide estimates of the means and standard deviations.

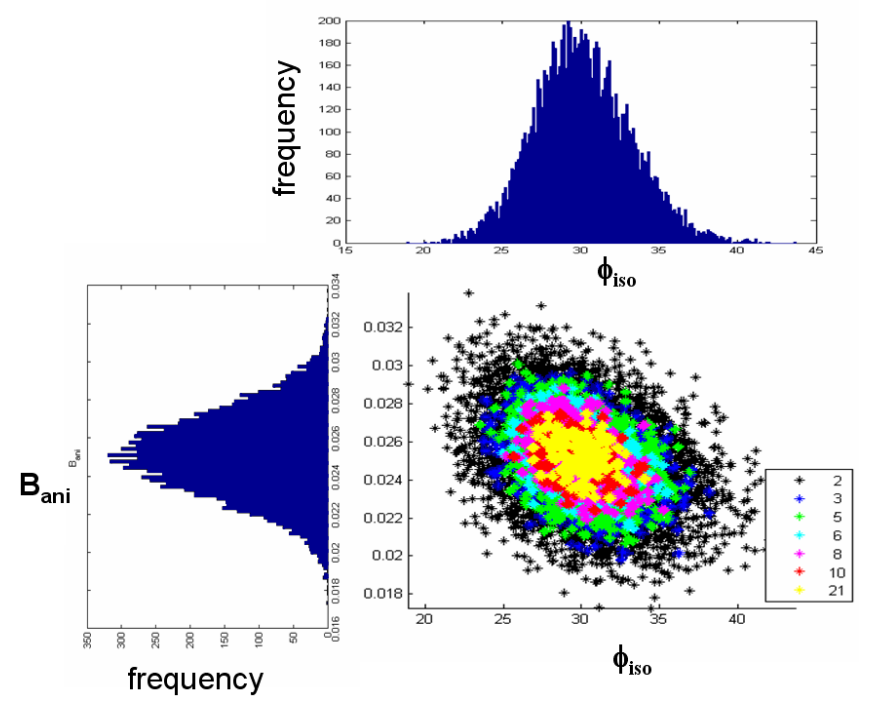

Figure 3. Example distributions used for estimating $B_{a n i}, \Phi_{i s o}, \sigma_{B_{a n i}}$ and $\sigma_{\Phi_{i s o}}$.

The results of this process provide a 3D volume for the two parameters and their respective uncertainty: $B_{a n i}, \sigma_{B_{a n i}}, \Phi_{i s o}$, and $\sigma_{\Phi_{i s o}}$. The standard volume mapping is the horizontal dimensions corresponding to space, and the vertical dimension 


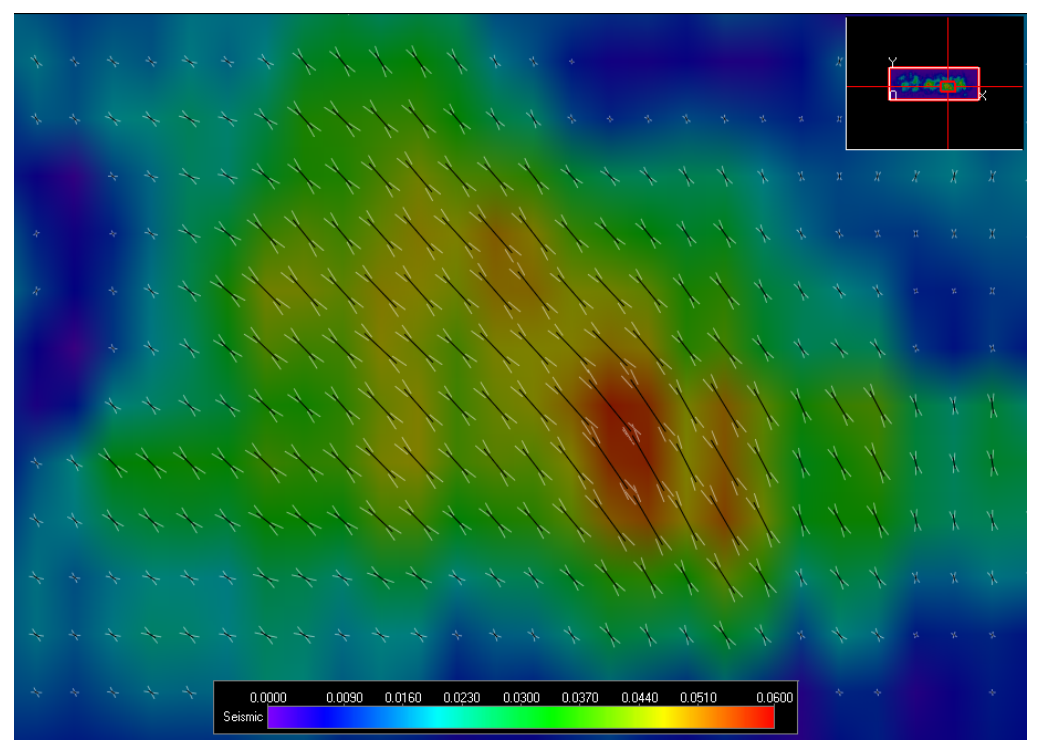

Figure 4. Static glyph illustrating magnitude and orientation along with uncertainty in each component. This can be compared against the colour mapped slice showing only $B_{a n i}$.

representing time (further stages in processing can map the wave related time dimension to space/depth). The voxels are also highly anisotropic in that the time resolution is greater than the spatial resolution. This not to be confused with the rock anisotropy property $B_{a n i}$ that is being computed. Thus, while these volumes could be visualized using volume rendering schemes, they have high frequencies in the time dimension which complicates interpretation. Therefore, 2D slice planes are traditionally the preferred visualization. The approximation of the integrals (Equations 1 and 2) is also done on a time slice basis and the resulting vectors all lie on the time plane. The result is a stack of $2 \mathrm{D}$ bidirectional vector fields and their associated uncertainty.

Bidirectional vector fields add uncertainty about the sign of a vector. While directional vector fields can be thought of as vectors starting at locations on a grid, bidirectional vector fields can be imagined as line segments centered on grid cells. Therefore unique orientations are only in the range of 0 to $\pi$ radians (for the $2 \mathrm{D}$ case).

\section{VISUALIZATIONS OF BIDIRECTIONAL VECTOR FIELDS}

We will describe our implementation of both a glyph-based and animated flow-based visualization of this data. Each visualization fills a different niche, but they can also be utilized together.

\subsection{Glyph-based Representation}

Wittenbrink et al.'s ${ }^{23}$ static glyph provided a compact representation of the information allowing for specific realizations to be imagined within the bounds of the glyph itself. Their glyph however could begin to look cluttered with highly dense fields and large angular uncertainty (greater than \pm 45 degrees), and would have become even more congested with bidirectionality. Our glyphs provide for dense fields while still maintaining readability. The glyphs can be displayed on the currently selected horizontal slice plane, tracking along a surface, or throughout the entire 3D volume. Displaying a dense field was the main use case and so "minimizing data-ink" 20 was a useful design principle as also considered by Wittenbrink et al. ${ }^{23}$ This principle can also be considered as a heuristic for evaluation ${ }^{27}$ and has been applied to analyze uncertainty visualizations. ${ }^{24}$

\subsubsection{Implementation}

Various viewing points and navigations are common for interpretation of the data in relation to other structures, such as geological surfaces and well core data. Therefore the glyph would potentially be viewed from all angles. While we considered and prototyped multiple glyphs the final version that shows both magnitude and orientation and their uncertainty 


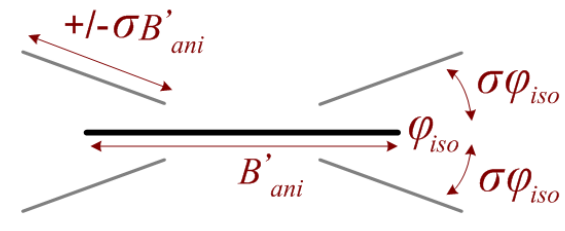

TOP VIEW

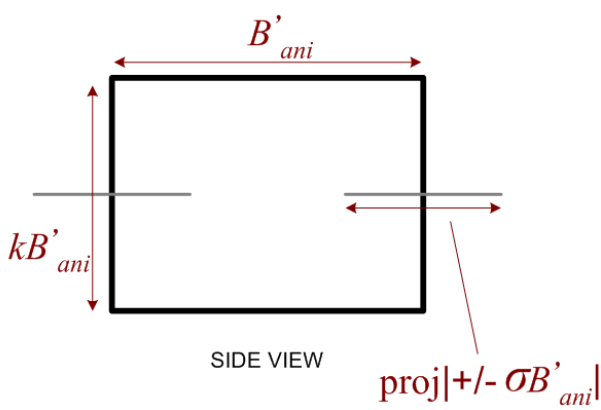

Figure 5. Static glyph illustrating bidirectional magnitude and orientation along with uncertainty in each component. $B_{a n i}^{\prime}$ represents a user controlled mapping to length of the dimensionless $B_{a n i}$ in the form $k_{1} B_{a n i}^{k 2}$.

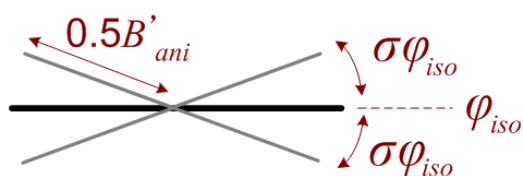

TOP VIEW

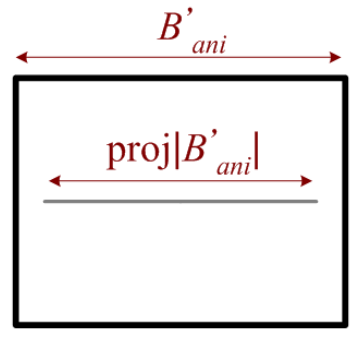

SIDE VIEW

Figure 6. Static glyph illustrating bidirectional magnitude and orientation along with uncertainty in orientation only.

is shown in Figures 1 and 4, and in diagram form in Figure 5. The rectangle is used to encode $B_{a n i}$ and $\Phi_{i s o}$ while the less strong lines reveal the uncertainty in both. For visualization of uncertainty only in orientation an additional glyph was created to simplify the reading as shown in diagram form in Figure 6 and in a visualization in Figure 7.

Both glyphs were designed to provide the clearest reading of all encodings at the top down viewing angle, but also allow reading of the orientation and magnitude at various oblique angles. As noted in the diagrams the side views (and all non-top views) show some projection of $\sigma_{B_{a n i}}$ but this is also true for any non perpendicular view of $B_{a n i}$. The magnitude of $B_{a n i}$ is encoded in both the length of the rectangle and the height. This design prioritizes occlusion in a dense field based on $B_{a n i}$ and thereby overall trends can be observed even when the viewpoint is close to the slice plane as seen in Figure 1 . In the overhead views of the second glyph the magnitude of $\sigma_{\Phi_{i s o}}$ will draw attention at large values, while at small values it tends to accentuate the edge detection of the darker line segment as shown in Figure 7.

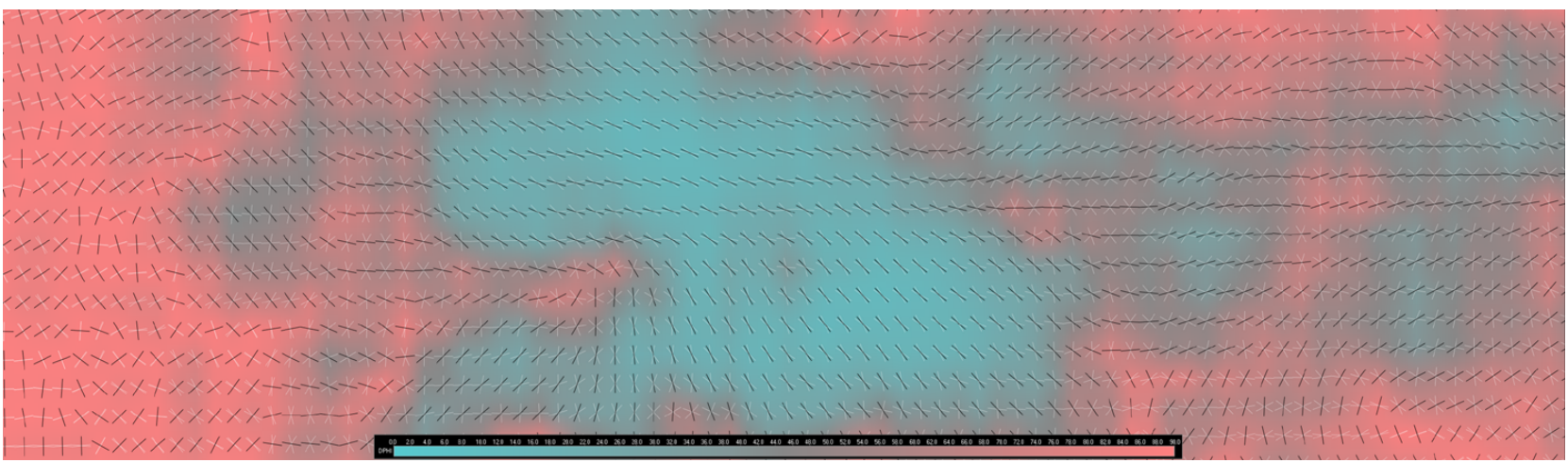

Figure 7. Use of the glyph depicting uncertainty in orientation only for inspection of a large field (quality control of orientation field). The colour mapping on the slice redundantly shows orientation uncertainty $\sigma_{\Phi_{i s o}}$. 


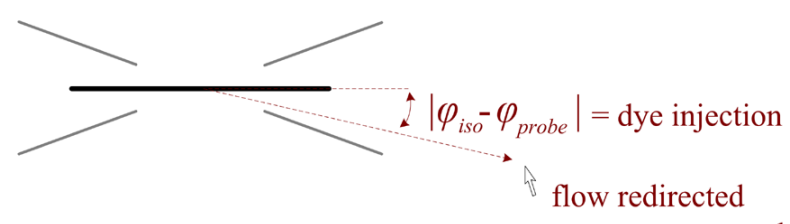

TOP VIEW on vector to cursor probe

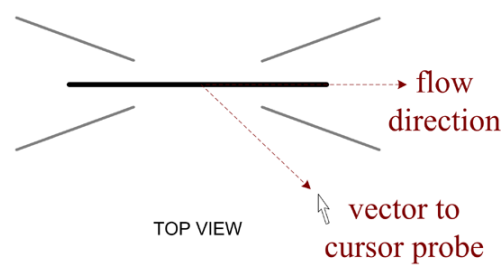

Figure 8. Left diagram: Flow redirection and calculation of uncertainty feedback for dye injection. Right diagram: No reorientation when query direction is beyond $\sigma_{\Phi_{i s o}}$.

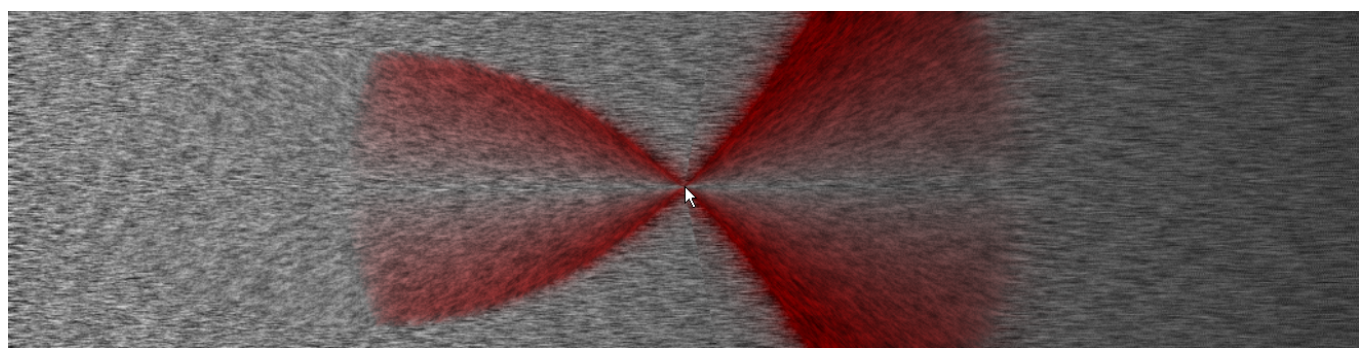

Figure 9. Illustration of flow reversal and reorientation query. Initial vectors are horizontal (flow left to right) with angular uncertainty increasing from 0 at the left edge of image to \pm 90 degrees at the right. Red dye shows amount of angular reorientation in redirected flow toward cursor.

\subsubsection{Interaction with the representation}

The visual appearance of a field of glyphs is quite different from individual glyphs. Therefore we provide interactive manipulation of the mapping of $B_{a n i}$ to glyph length (both scalar and exponent factors, i.e. $k_{1} B_{a n i}^{k 2}$ ) to create various overall field visualization effects. Another scalar $k$ provides additional glyph height manipulation to provide the user control over the occlusion possible from various viewing orientations. If the size of a glyph is scaled beyond the size of a single voxel they may overlap and appear as a hatching style, thus creating a new regional representation.

The glyph in its natural form emphasizes uncertainty as it enlarges based on the uncertainty. Switching between the two glyphs in Figures 5 and 6 allows one to vary the focus on the uncertainty. In the first the $\sigma_{B_{a n i}}$ is an overriding factor of emphasis as it affects the viewing size, thus with very small $\sigma_{B_{a n i}}$ the uncertainty components may be difficult to perceive even with large $\sigma_{\Phi_{i s o}}$. While in the second glyph only $\sigma_{\Phi_{i s o}}$ has a role and so is useful for considering angular uncertainty in isolation, due to the possible dominant reading of $B_{a n i}$ in the first glyph (reading of length vs. orientation).

\subsection{Flow-based Representation}

Recently Botchen et al. ${ }^{3}$ presented three advection approaches of multi-frequency noise, cross advection, and Gaussian error diffusion, for showing uncertainty in flow fields. Taking a different approach to revealing uncertainty as a diffusionlike process, we allow user queries using a cursor probe to reverse and reorient flow vectors within the angular uncertainty $\left(\sigma_{\Phi_{i s o}}\right)$ and visually reveal the magnitude of reorientation with the amount of coloured dye injection. With this new approach the user can see directly using an interactive query rather than having to internally imagine variation in flow. The user can thus create a simple form of unsteady flow ${ }^{4}$ based on their interaction via the cursor probe.

In a pre-processing step the ambiguity of bidirectionality should be resolved to one preferential direction. This should be done based on a spatially consistent scheme, or it could be assigned the most probable direction. These flow vectors can then be reversed and reoriented based on the position of the cursor probe. This process is explained in Figure 8 and for illustrative purposes we show the effects on simple left to right flow with increasing angular uncertainty (from 0 to \pm 90 degrees) in Figure 9. The distance over which the cursor probe affects flow is set by the user. Reversals are not considered reorientation for the calculation of the amount of dye injection, due to the directional ambiguity.

This flow simulation is then used as an animated texture on the corresponding horizontal slice plane of the 3D volume. The user may interact with the slice with a probe or change visualization parameters, and can drag the slice plane up and down in the volume where the corresponding flow visualization will be started. 


\subsubsection{Implementation}

With a GPU-based implementation we use textures to store our vector field variables $\left(B_{a n i}, \sigma_{B_{a n i}}, \Phi_{i s o}\right.$, and $\left.\sigma_{\Phi_{i s o}}\right)$ and a GLSL fragment program to perform the flow advection. Spot noise textures are also utilized to create the streak-lines as described by van Wijk. ${ }^{21}$ The flow simulation state for the current and previous time step are held in two OpenGL framebuffer objects (FBO). The previous time step FBO is used as a texture source and the current FBO as a rendering target to avoid the need for any OpenGL context switching. With this scheme the fragment program uses multitexturing to access all required grid data for the visualization.

Without coordinate variable representations fragment programs compute regularly spaced grid output. Thus our simulation steps backward in time $\left(t_{n-1}\right)$ to advect toward the grid cell centers at the current time $\left(t_{n}\right)$, rather than forward advecting results from the grid centers. This general scheme has been used by Botchen et al. ${ }^{3}$ and Jobard et al. ${ }^{8}$ Our advection vector $\left(\vec{v}_{x}\right)$ is computed as a function of location $(x=(i, j))$, all vector field variables, and the cursor probe location $\left(x_{\text {probe }}\right)$, at the current time $\left(t_{n}\right)$ as,

$$
\vec{v}_{x}\left(x, t_{n}\right)=s_{x}\left(x, B_{a n i}(x), \sigma_{B_{a n i}}(x), t_{n}\right) \vec{d}_{x}\left(x, x_{\text {probe }}, \Phi_{i s o}(x), \sigma_{\Phi_{i s o}}(x)\right) \Delta t,
$$

with the function $s_{x}$ being a pseudo-speed function, and function $\vec{d}_{x}$ providing a normalized direction vector. $\vec{d}_{x}$ is computed as either $\Phi_{i s o},-\Phi_{i s o}$, or the direction to the probe $\left(x_{\text {probe }}-x\right)$ depending on its proximity to the probe being less than the user specified threshold and the angular difference between this direction and $\Phi_{i s o}(x)$ being less than $\sigma_{\Phi_{i s o}}(x)$. The flow speed $s_{x}$ is a function of $B_{a n i}$ computed using user specified constants $k_{1}$ and $k_{2}$ as $k_{1} B_{a n i}^{k 2}$. The user also has the option for viewing the flow animated over the domain $\left(B_{a n i} \pm \sigma_{B_{a n i}}\right)$. For this $s_{x}$ animation the values oscillate with linear interpolation between \pm one standard deviation over a fixed number of interpolation steps, but using delays computed from a Gaussian function for each step. Thus the flow duration for any given speed varies between a user specified duration of $T_{\text {user }}$ at $B_{\text {ani }}$ to $\frac{1}{e} T_{\text {user }}$ at the extreme values $\left(B_{a n i} \pm \sigma_{B_{a n i}}\right)$. Calculation of the current grid location at our current time step is performed with the equation:

$$
f\left(x, t_{n}\right)=f\left(x-\vec{v}_{x}, t_{n-1}\right)+g\left(x, x_{\text {probe }}, \Phi_{\text {iso }}(x), \sigma_{\Phi_{\text {iso }}}(x)\right),
$$

where the grid $f$ forms the final texture image, and $g$ is any dye-like contribution (including spot noise). Uncertainty in magnitude is encoded with the $s_{x}$ animation that is reflected in $\vec{v}_{x}$. The calculation of function $g$ includes a linear blend of the spot-noise and a colour which reveals uncertainty in direction. The blend being proportional to the magnitude of the flow redirection. This is not an overlay but modification of the grid cell (RGB texture) contents which are advected. The repeated evaluation of $f$ can be considered an Euler Forward Method integration of the flow simulation. Use of the non-grid aligned previous time step results (i.e. $f\left(x-\vec{v}_{x}, t_{n-1}\right)$ ) is performed using bilinear texture filtering. The noise injection creates the variations of streak-lines based on the user controlled blending rate of previous time step results.

Our visualization does not represent actual flow velocities but only is intended to provide relative comparisons between areas. For our use of "flow" only as a graphic variable, the limiting of velocity (and thus advection distance) to around one grid cell width is acceptable, thus maintaining the Courant-Friedrichs-Lewy Condition (numerical stability) and avoiding aliasing of the spot-noise patterns. Therefore we scale the visualization flow velocities down to a unit range, which is equivalent to reducing the flow simulation time step, to avoid these same issues. This enables us to skip any post advection filtering step as used by Jobard et al. ${ }^{8}$ If the CPU or graphics hardware cannot attain the user requested animation framerate the net animation velocities are effectively slowed down as well. The actual velocities, which would be slow and whose estimation would be extremely approximate, along with the scale of data (on the range of kilometres) preclude the value of a temporally realistic simulation. More importantly velocity is only related to $B_{\text {ani }}$. However, flow is a good graphical encoding as it relates to an actual realization of interest. The majority of the computation time is spent in the fragment program calculating Equations 3 and 4 (direction determination, Euler integration, dye additions).

\subsubsection{Interaction with the representation}

With the flow visualization we allow the user to interactively reverse local flow by moving the cursor probe, as either a sink or source, over the field. Within a user defined distance of the sink cursor probe, any vector that points away from the cursor is automatically flipped and vice versa for the source. In the application domain this may correspond with reality in that an oil field well may either pump in fluids or be used for extraction.

Similarly the user can also explore explicit realizations with the cursor probe as flow vectors are reoriented directly toward the cursor if this new vector lies within their orientation uncertainty as defined by $\sigma_{\Phi_{i s o}}$. Our use of colour specifically 


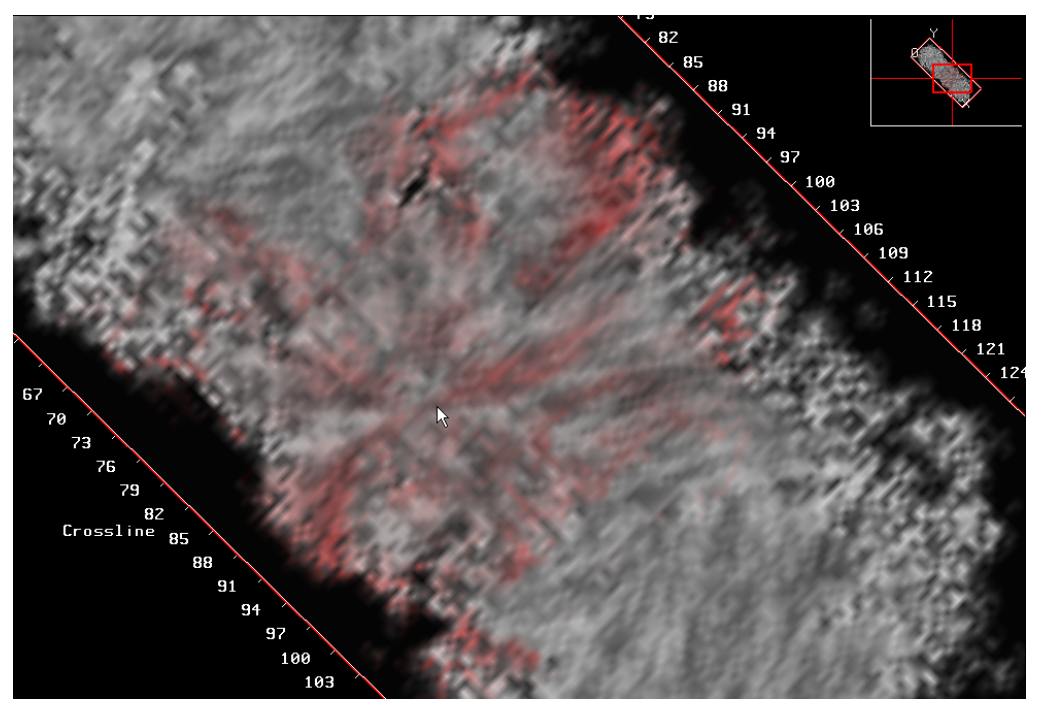

Figure 10. Animation frame showing user flow query. Red dye injection is based on the difference between user requested flow orientation (directly to cursor) and the most likely direction.

provides visual feedback indicating the difference between the most likely orientation of each vector and the user requested orientation. The results of this interaction are shown in Figure 10.

Opposite to the glyph, when there is no user interaction the uncertainty in the flow visualization is de-emphasized. Selective emphasis of uncertainty is provided based on the user's movement of the cursor probe revealing uncertainty in flow direction. The uncertainty is injected like a dye and then flows along streamlines fading with the spot-noise.

Within the flow, user thresholding of regions is also performed by blending highly uncertain areas to black. This is currently based on a lower threshold of $B_{a n i}$ as it is correlated with $\Phi_{i s o}$ in that orientation can not be defined at low levels of anisotropy. Thus the user can eliminate areas from consideration; this helps the user to avoid watching for patterns in regions of arbitrarily assigned directions.

\section{VISUALIZATION USE}

These visualizations may be used to interactively explore the data and its uncertainty on one slice plane at a time. The previously described user controllable variations allow their use to be tailored to the specific phase of interpretation and exploration.

\subsection{Glyph and Flow Integration}

The user can choose to combine both the flow visualization and the static glyphs. This allows the unique benefits of each to be combined. The user can be guided by the reference provided by one visualization style while interactively adjusting the display parameters of the other. The movement provided by the flow may also enhance visualization with large displays by utilizing the stronger perception of motion in peripheral vision. ${ }^{22}$ Figure 11 shows the glyph on its own and then combined with the flow visualization, both from a more distant viewing point where the uncertainty encodings are deemphasized.

\subsection{Simplified Visualizations}

All parameters including uncertainty can be viewed as standard colour mapped slice planes through the volume, as shown in Figure 7. This allows the uncertainty to be treated as data rather than meta-data and simplifies tasks that may only concern uncertainty issues. The second glyph representation, that was shown in Figure 6, also provides a form of this in that it allows a simpler reading of the orientation uncertainty on its own as $B_{a n i}^{\prime}$ can be chosen to be replaced with a unit sized vector (example shown in Figure 7). This is important as when considering uncertainty information it is helpful to allow the user to decompose and view each component individually to assist in comprehension or during interpretation. 


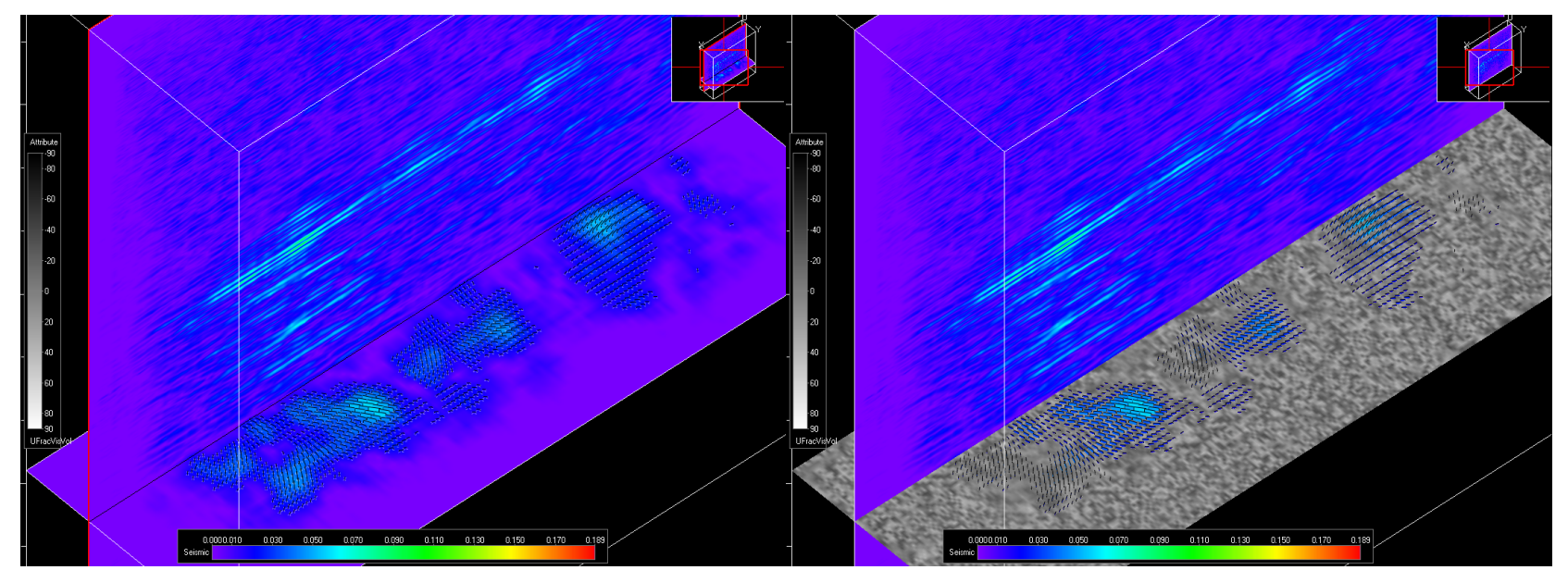

Figure 11. Distant views of glyph alone (left image) and with flow visualization (right image) along one slice of $3 \mathrm{D}$ volume of data. Vertical slice shows the relatively higher frequency data in that dimension.

\subsection{Tasks}

Returning to consider the tasks used by Laidlaw et al.'s ${ }^{10}$ evaluation (locating critical points, identifying their type, and predicting particle advection), our animated flow visualization may aid in locating critical points as the motion may enlarge the user's useful field of view. ${ }^{22}$ However an evaluation would be required to determine how easily the motion of specific types of critical points can be perceived, preattentively or otherwise, with the other flow motions within this larger field of view. Additionally for both the tasks of identifying the type of critical point and particle advection prediction, the explicit streamline tracing and unambiguous flow direction should be of assistance.

The bidirectionality is important to explore in the seismic industry, and as stated previously new sources or sinks (which would be wells) can be interactively placed to visualize the resulting flow as vectors are reversed and redirected. Uncertainty in the amount of redirected flow was made apparent by the amount of red dye being injected and transported. Bidirectionality of the vectors was also inherent when reading the glyphs, and overall trends may be seen with both the flow and glyph visualizations individually or combined.

\section{CONCLUSION}

We have created two new and differently styled visualizations for uncertainty in bidirectional vector fields. These two new visualizations extended previous work in the area of uncertainty visualization and vector fields for both bidirectionality of the vectors and richness of interactivity. The user-controllable aspects of the glyph and flow allow visualizations that respectively vary from precise, micro reading in an abstract form, to macro reading closer to actual realizations of interest in the seismic domain.

With regard to future work, while these visualizations focused on the data uncertainty, it may also be worth considering visualizing the uncertainty in interpretation. For example, if the critical points in possible flow fields were automatically detected, such as with Ford's approach, ${ }^{6}$ they could be labelled showing the classification confidence. We expect it may be of value to integrate this or the interpreter's confidence directly into the visualization as a decision aid. ${ }^{26}$

\section{REFERENCES}

1. R. Amar and J. Stasko. Knowledge precepts for design and evaluation of information visualizations. Visualization and Computer Graphics, IEEE Transactions on, 11(4):432-442, 2005.

2. M. K. Beard and B. Buttenfield. Detecting and evaluating errors by graphical methods. In P. Longley, M. Goodchild, D. Maguire, and D. Rhind, editors, Geographic Information Systems: Principles and Technical Issues. Vol. 1, pages 219-233. John Wiley, New York, 1999.

3. R. P. Botchen, D. Weiskopf, and T. Ertl. Texture-based visualization of uncertainty in flow fields. In Visualization, 2005. VIS 05. IEEE, pages 647-654, 2005. 
4. K. Bürger, J. Schneider, P. Kondratieva, J. Krüger, and R. Westermann. Interactive visual exploration of unsteady $3 \mathrm{~d}$ flows. In Proceedings of Eurographics /IEEE VGTC Symposium on Visualization (EuroVis 2007, May 23-25, 2007, Norrköping, Sweden), Eurographics Workshop Series, Aire-la-Ville, 2007. Eurographics.

5. J. Downton and D. Gray. AVAZ parameter uncertainty estimation. In 76th Annual International Meeting, SEG, Expanded Abstracts, pages 234-238, 2006.

6. R. M. Ford. Critical point detection in fluid flow images using dynamical system properties. Pattern Recognition, 30(12):1991-2000, 1997.

7. G. Grigoryan and P. Rheingans. Point-based probabilistic surfaces to show surface uncertainty. IEEE Transactions on Visualization and Computer Graphics, 10(5):564-573, 2004.

8. B. Jobard, G. Erlebacher, and M. Y. Hussaini. Lagrangian-eulerian advection of noise and dye textures for unsteady flow visualization. IEEE Transactions on Visualization and Computer Graphics, 8(3):211-222, JUL/SEP 2002.

9. C. Johnson. Top scientific visualization research problems. IEEE Computer Graphics and Applications, 24(4):13-17, July/August 2004.

10. D. Laidlaw, R. Kirby, C. Jackson, J. Davidson, T. Miller, M. da Silva, W. Warren, and M. Tarr. Comparing 2d vector field visualization methods: a user study. Visualization and Computer Graphics, IEEE Transactions on, 11(1):59-70, Jan/Feb 2005.

11. S. K. Lodha, A. Pang, R. E. Sheehan, and C. M. Wittenbrink. UFLOW: Visualizing uncertainty in fluid flow. In R. Yagel and G. M. Nielson, editors, IEEE Visualization '96, pages 249-254. IEEE, 1996.

12. A. L. Love, A. Pang, and D. Kao. Visualizing spatial multivalue data. IEEE Computer Graphics and Applications, 25(3):69-79, May/June 2005.

13. A. M. MacEachren. Visualizing uncertain information. Cartographic Perspective, 13:10-19, 1992.

14. A. M. MacEachren, A. Robinson, S. Hopper, S. Gardner, and R. Murray. Visualizing geospatial information uncertainty: What we know and what we need to know. Cartography and Geographic Information Science, 32(3):139-160, 2005.

15. A. T. Pang, C. M. Wittenbrink, and S. K. Lodha. Approaches to uncertainty visualization. The Visual Computer, 13(8):370-390, 1997. ISSN 0178-2789.

16. P. Rheingans and S. Joshi. Visualization of molecules with positional uncertainty. In E. Gröller, H. Löffelmann, and W. Ribarsky, editors, Data Visualization '99, pages 299-306. Springer-Verlag Wien, 1999.

17. A. R. Sanderson, C. R. Johnson, and R. M. Kirby. Display of vector fields using a reaction-diffusion model. In Visualization, 2004. IEEE, pages 115-122, 2004.

18. A. Telea and R. Strzodka. Multiscale image based flow visualization. In R. F. Erbacher, J. C. Roberts, M. T. Gröhn, and K. Börner, editors, Proc. SPIE \& IS\&T Conf. Electronic Imaging, Vol. 6060: Visualization and Data Analysis 2006, 2006. 606001.

19. J. Thomson, E. Hetzler, A. MacEachren, M. Gahegan, and M. Pavel. A typology for visualizing uncertainty. In Proc. SPIE \& IS\&T Conf. Electronic Imaging, Vol. 5669: Visualization and Data Analysis 2005, pages 146-157, 2005.

20. E. R. Tufte. The Visual Display of Quantitative Information. Graphics Press, Cheshire, CT, 2nd edition, 2001.

21. J. J. van Wijk. Image based flow visualization. ACM Transactions on Graphics (TOG), 21(3):745-754, 2002.

22. C. Ware. Information Visualization: Perception for Design. Morgan Kaufmann Publishers, 2nd edition, 2004.

23. C. M. Wittenbrink, A. T. Pang, and S. K. Lodha. Glyphs for visualizing uncertainty in vector fields. IEEE Transactions on Visualization and Computer Graphics, 2(3):266-279, 1996.

24. T. Zuk and M. S. T. Carpendale. Theoretical analysis of uncertainty visualizations. In R. F. Erbacher, J. C. Roberts, M. T. Gröhn, and K. Börner, editors, Proc. SPIE \& IS\&T Conf. Electronic Imaging, Vol. 6060: Visualization and Data Analysis 2006, 2006. 606007.

25. T. Zuk, M. S. T. Carpendale, and W. Glanzman. Visualizing temporal uncertainty in 3D virtual reconstructions. In Proc. of the 6th International Symposium on Virtual Reality, Archaeology and Cultural Heritage (VAST 2005), pages 99-106, 2005.

26. T. Zuk and S. Carpendale. Visualization of uncertainty and reasoning. In Proceedings of the 7th International Symposium on Smart Graphics (June 25-27, 2007, Kyoto, Japan), Berlin, Heidelberg., 2007.

27. T. Zuk, L. Schlesier, P. Neumann, M. S. Hancock, and S. Carpendale. Heuristics for information visualization evaluation. In Proceedings of the 2006 AVI workshop on BEyond time and errors: Novel Evaluation Methods for Information Visualization (BELIV 2006), pages 55-60. ACM Press, 2006. 